\title{
ANÁLISE DA PRODUÇÃO CIENTÍFICA DOS ENFERMEIROS DE MINAS GERAIS PUBLICADA EM PERIÓDICOS DE ENFERMAGEM ${ }^{1}$
}

Roberta dos Santos Costa

Daclé Vilma Carvalho ${ }^{3}$

Costa RS, Carvalho DV. Análise da produção científica dos enfermeiros de Minas Gerais publicada em periódicos de enfermagem. Rev Latinoam Enfermagem 2001 setembro-outubro; 9(5):19-25.

Trata-se de um estudo descritivo, exploratório e retrospectivo sobre a produção cientifica dos enfermeiros de Minas Gerais publicada em periódicos de enfermagem do BDENF, de 1989 a 1998. Teve como objetivo analisar a produção científica dos enfermeiros mineiros em periódicos nacionais de enfermagem que compõem o BDENF, 5613 nomes de enfermeiros cadastrados no COREn/MG, foram lançados neste banco, com a intenção de encontrar os autores e suas produções. Encontramos 54 autores e 87 publicações e a média de publicação por autor foi de 1,6. Os autores e co-autores que produzem e divulgam são docentes, com seus pares de titulação.

PALAVRAS CHAVE: bancos de dados, revistas, enfermagem

\section{ANALYSIS OF THE SCIENTIFIC PRODUCTION BY NURSES FROM MINAS GERAIS PUBLISHED IN NURSING JOURNALS}

This is a descriptive, exploratory and retrospective study on the scientific production by nurses from Minas Gerais in BDENF Nursing journals from 1989 to 1998. The main objective was to characterize the scientific production by nurses from Minas Gerais published in the national nursing journals which comprise the BDENF. The names of 5.613 nurses from the records of COREn/MG were included in the database in order to find authors and their writings. Fifty-four authors and 87 writings were found with an average of 1.6 writings per author. The authors and co-authors who produce and publish are university professors working in collaboration with their peers.

\section{ANÁLISIS DE LA PRODUCCIÓN CIENTÍFICA DE LOS ENFERMEROS DE MINAS GERAIS PUBLICADA EN PERIÓDICOS DE ENFERMERÍA}

Se trata de un estudio descriptivo, exploratorio y retrospectivo sobre la producción cientifica de los enfermeros del Estado de Minas Gerais en Revistas nacionales de enfermería que componen. el (banco de datos de enfermería). BDENF. 5613 nombres de enfermeros registrados en el Consejo Regional De Enfermería/Minas Gerais - COREn/MG, fueron colocados en éste banco, con la intención de encontrar Ios autores y su producción. Encontramos 54 autores y 87 publicaciones, el promedio de publicación por fue de 1,6. Los autores y coautores que producen y divulgan son docentes, con sus pares de titulación.

PALABRAS CLAVES: banco de datos, revista, enfermería

\footnotetext{
${ }^{1}$ Texto elaborado a partir da dissertação apresentada à Escola de Enfermagem da Universidade Federal de Minas Gerais, para obtenção de título de Mestre em Enfermagem. ${ }^{2}$ Enfermeira. Mestre, Professor da Escola de Auxiliar de Enfermagem João Paulo II, e-mail: Rbm@mkm.com.br; ${ }^{3}$ Enfermeira. Doutor, Professor da Escola de Enfermagem da Universidade Federal de Minas Gerais (Orientadora). e-mail: dacle@enf.ufmg.br
} 
INTRODUÇÃO

A pesquisa em enfermagem é importante porque legítima o fazer, busca novas formas de cuidar, aproxima as dimensões teóricas e práticas do trabalho de enfermagem, contribui para a qualidade de vida da população, produz conhecimento e dá sustentação à prática de enfermagem ${ }^{(1)}$.

Assim podemos dizer que quanto mais dúvidas maior as possibilidades de novas descobertas e conseqüentemente de novos conhecimentos e quanto mais se aprende menos se sabe e que respostas obtidas levantam mais sugestões ${ }^{(2)}$.

O desenvolvimento do conhecimento científico é muito rápido, conseqüentemente dinâmico e retrata o nível intelectual $e$ profissional em uma dada região.

Em levantamento bibliográfico sobre a produção científica de enfermagem encontramos análise da mesma nos Estados de São Paulo, Rio Grande do Sul, Santa Catarina e outros, revelando que o Estado de Minas Gerais, apesar de ter a segunda maior população do país, contar com 853 municípios, ser a segunda economia brasileira, e possuir 12 escolas de enfermagem e 5.664 enfermeiros cadastrados no Conselho Regional de Enfermagem até novembro de 1999, ainda não possuía um levantamento e análise sobre a produção científica dos seus enfermeiros.

Assim, buscando respostas para as indagações: Quais são as características da produção científica dos enfermeiros do Estado de Minas Gerais divulgados em periódicos nacionais? E quem são os enfermeiros que estão divulgando seus trabalhos? Realizamos um estudo sobre a produção científica dos enfermeiros mineiros publicada em periódicos de enfermagem nacional.

Apesar de neste trabalho não termos tido a intenção de diagnosticar a situação do saber em enfermagem acreditamos que de algum modo diagnosticamos a situação das publicações no Estado de Minas Gerais.

\section{OBJETIVO}

Analisar a produção científica dos enfermeiros do Estado de Minas Gerais, publicada em periódicos de enfermagem nacionais que compõe o Banco de Dados de Enfermagem-BDENF.

\section{OBSTÁCULOS À DIVULGAÇÃO DO CONHECIMENTO EM ENFERMAGEM}

Todo conhecimento produzido necessita e deve ser transmitido a outras pessoas com o objetivo de levá-las a pensar e a perceber tanto as coisas familiares de modo diferente, quanto obter novas informações. E uma das formas de se transmitir os conhecimentos é por meio da divulgação científica: um trabalho de pesquisa, seja ele documental, bibliográfico ou de campo, deve ser publicado para que seus resultados sejam reconhecidos no meio cientifico ${ }^{(3)}$. Em qualquer área profissional, inclusive a enfermagem, a divulgação é importante pois, é graças a novas informações que aprendemos. A comunicação dos resultados da pesquisa permite que outros profissionais tenham a oportunidade de conhecer, avaliar e questionar problemas que eventualmente possam surgir sobre as questões estudadas.

Porém, vários são os obstáculos que interferem e dificultam a comunicação dos conhecimentos. 0 primeiro é a obtenção de espaços para a publicação das pesquisas nos periódicos. 0 segundo é o comportamento do usuário de não possuir o hábito de leitura ou não utilizar as bibliotecas ou outras fontes de pesquisa. E o terceiro obstáculo é a demora dos pesquisadores em publicar os resultados de suas pesquisas. Estes obstáculos podem levar a repetição desnecessária de temas de pesquisas e impedir a continuidade ou 0 aprofundamento dos mesmos ${ }^{(4)}$.

A pesquisa de enfermagem e sua divulgação vêm evoluindo no Brasil de forma lenta e gradual ${ }^{(5)}$ e talvez esta lenta difusão do conhecimento seja decorrente dos canais utilizados pelos enfermeiros ${ }^{(6)}$. Os canais mais utilizados para divulgação pelos enfermeiros na divulgação científica são os congressos, simpósios e reuniões científicas, sendo que o número de publicações em periódicos científicos ainda é reduzido ${ }^{(7-10)}$.

Outro meio de divulgação utilizado em menor escala pelos enfermeiros são os periódicos científicos, e que possuem algumas vantagens: publicação regular, podem estimular a produção de novos trabalhos e facilidade de arquivo e consulta ${ }^{(7)}$.

\section{MATERIAL E MÉTODO}

Esse estudo é descritivo, de caráter exploratório e retrospectivo. Optamos por trabalhar somente com periódicos de enfermagem nacional indexados no Banco de Dados de Enfermagem (BDENF), criado na Escola de Enfermagem da UFMG, em Belo Horizonte, e que tem como objetivos coletar, selecionar, armazenar e processar a literatura convencional e não convencional da área de enfermagem gerada no país. Até o dia 07 de abril de 2.000, esse banco de dados contava com 7.288 trabalhos registrados e 17 periódicos indexados. Os dados que se encontram no quadro abaixo fazem uma estimativa dos periódicos publicados desde sua criação e que compõem o BDENF. 
Takela 1 -Características, thlose estimativa de fascículos dos periódicos cadastrados no BDENF. Belo Horizonte, 2000

\begin{tabular}{|c|c|c|c|c|c|}
\hline \multirow[t]{2}{*}{ Thubdo Ferídico } & \multirow[t]{2}{*}{ Priodicitede } & \multirow[t]{2}{*}{$\begin{array}{l}\text { Preatimative de } \\
\text { tacicubo putic J10 }\end{array}$} & \multirow[t]{2}{*}{$\begin{array}{l}\text { Inćio de publicasăo } \\
\text { doe períbicoos }\end{array}$} & \multicolumn{2}{|c|}{$\begin{array}{l}\text { Totalde tacinderado日 na } \\
\text { bibl.compüsdagudude }\end{array}$} \\
\hline & & & & p & 4 \\
\hline $\begin{array}{l}\text { Rievistabirasileira deEnfermagem ibuligagăón } \\
\text { comen nté) }\end{array}$ & Trimestal & 172 & 1955 & 73 & $d \hat{z}$ \\
\hline $\begin{array}{l}\text { Reeviste de Esc. Enfermagem da UP } \\
\text { (publigağb comante) }\end{array}$ & gyadnim estral & $9 s$ & 1967 & 70 & 75 \\
\hline Enfoque (publicaça conente) & gyadnim estral & 96 & 1972 & 55 & $5 \pi$ \\
\hline $\begin{array}{l}\text { Enfemmagem Nouas dimensŏ́es ppublicaçăo } \\
\text { come nte) }\end{array}$ & Bimestal & 24 & 1975 & 28 & 96 \\
\hline 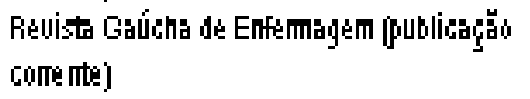 & Semestal & 44 & 1976 & 35 & 80 \\
\hline 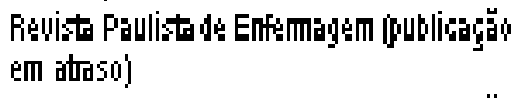 & Bimestral & 78 & 1981 & $\$ 0$ & $\$ 9$ \\
\hline $\begin{array}{l}\text { Reuista Baiana de Enfemmagem [publicaçăo } \\
\text { em atrasoj }\end{array}$ & Semestal & 24 & 1985 & 18 & 55 \\
\hline 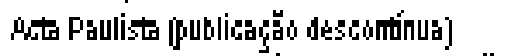 & Trimestal & 40 & 1988 & 30 & 75 \\
\hline 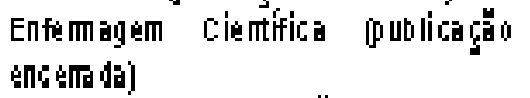 & Semestral & 4 & 1990 & 4 & 100 \\
\hline Texto e Contex to (bublicagăo gomente) & Semestal & 14 & $19 \dot{y} \hat{z}$ & 14 & 100 \\
\hline $\begin{array}{l}\text { Revista de Enfemagem da PUC (publicaçăo } \\
\text { em atrasoj }\end{array}$ & Semestal & 10 & 1998 & 7 & 60 \\
\hline 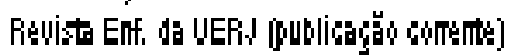 & Semestral & 10 & 1998 & 10 & 100 \\
\hline 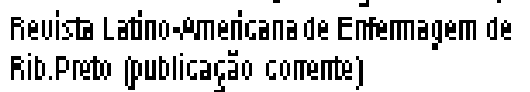 & Trimestral & 24 & 1998 & 17 & $\pi 1$ \\
\hline 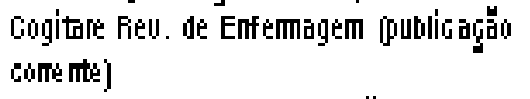 & semestal & 6 & 1996 & 5 & 80 \\
\hline Nursino êd. Brasileira ôublicactón comentel & Mensat & $\$ 6$ & 1986 & 5 & $1 \mathrm{~s}$ \\
\hline
\end{tabular}

A coleta de dados ocorreu entre os meses de agosto a dentre eles os que tinham publicações nos periódicos indexados. Após esta identificação, fizemos seleção das publicações que se enquadravam no período de estudo (1989-1998). Juntamente com a identificação do autor, obtivemos também o título do trabalho e informações sobre o periódico no qual este se localizava, ou seja, o volume, número, ano e páginas. Posteriormente, estes textos eram solicitados na biblioteca do campus da saúde ou pela BIREME, para análise.

De posse dos textos foram feitos a leitura e o registro das Enfermagem, criada em 1997 na Escola de Enfermagem da UFMG.

Para efeito de estudo, os trabalhos publicados por enfermeiros do Estado de Minas Gerais foram classificados em seis áreas temáticas ${ }^{(8)}$ : Assistencial, Administração em Enfermagem, Ensino, Profissional, Reflexões Teóricas e Outros. Na categoria outros foram agrupados trabalhos que não se enquadram nas anteriores.

Através de 3 formulários de coleta de dados adaptado de alguns autores ${ }^{(8-9)}$ categorizou os periódicos de enfermagem nacionais indexados no BDENF, bem como informações dos autores e coautores.

Os 5613 nomes dos enfermeiros cadastrados no COREn/ MG até 0 ano de 1998, foram lançados no BDENF, para identificar informações nos formulários, e os dados colhidos alimentavam um outro banco de dados criado especialmente para este estudo.

Foram detectados 54 (1\%) profissionais, que publicaram 87 trabalhos no período de 1989 a 1998, sendo a média de publicação por autor de 1,6. Destes, dois trabalhos não foram localizados na biblioteca da BIREME. Assim, foram analisados 85 trabalhos. Com relação aos autores desses dois trabalhos, apenas um foi excluído do estudo, já que o outro tinha outros trabalhos publicados. Portanto, foram analisados 53 autores e 85 trabalhos.

Aventamos ainda a possibilidade de terem ocorrido perdas de autores e conseqüentemente de trabalhos publicados, pois 
acreditamos que alguns deles não tenham sido identificados devido à não atualização de seus nomes no COREn/MG, nos casos de alteração dos mesmos.

Foram considerados enfermeiros mineiros aqueles que estão cadastrados no COREn/MG. Nos casos em que aparecem trabalhos com vários autores, a escolha do autor recaiu no primeiro nome citado no trabalho e como co-autor o segundo, com confirmação desses nomes na listagem do COREn/MG. Os demais nomes citados não foram considerados para análise.

Reafirmamos que os resultados aqui apresentados referemse somente aos fascículos indexados no BDENF, não significando portanto que esta pesquisa tenha abrangido a totalidade dos trabalhos publicados por enfermeiros mineiros no período estudado.

\section{APRESENTAÇÃO, ANÁLISE E DISCUSSÃO DOS RESULTADOS}

Foram encontradas publicações em 10 dos 17 periódicos analisados (59\%). Nestes, a Revista de Enfermagem da PUC/MG foi a que publicou o maior número de trabalhos de enfermeiros mineiros (32\%), seguida da Revista Mineira de Enfermagem, com 17 (20\%). Este fato pode ser justificado por serem periódicos do Estado de Minas Gerais, o que incentiva a publicação dos enfermeiros mineiros nos mesmos, e por serem reconhecidos no meio científico.

Na tabela 2 estão apresentados os 10 periódicos, nos quais foram encontrados publicações de enfermeiros mineiros, 0 ano e 0 número de publicações.

Tabela 2 - Periódicos de enfermagem segundo o ano de publicação no período de 1989 a 1998. Belo Horizonte, 2000

\begin{tabular}{|c|c|c|c|c|c|c|c|c|c|c|c|c|}
\hline Periódico & 89 & 90 & 91 & 92 & 93 & 94 & 95 & 96 & 97 & 98 & Total & $\%$ \\
\hline $\begin{array}{l}\text { Revista Enf. - Cademos Enf. - PUC-MG } \\
\text { Revista Mineira Enfermagem }\end{array}$ & & & & & 6 & 2 & 4 & 7 & $\begin{array}{c}2 \\
10\end{array}$ & $\begin{array}{l}6 \\
7\end{array}$ & $\begin{array}{l}27 \\
17\end{array}$ & $\begin{array}{l}32 \\
20\end{array}$ \\
\hline Revista Bras. de Enfermagem & 1 & - & - & 1 & 1 & - & 4 & 6 & - & - & 13 & 15 \\
\hline Revista da Escola de Enfermagem USP & - & 1 & 1 & - & - & 3 & 2 & 1 & 3 & - & 11 & 13 \\
\hline Revista Latino-Am. de Enfermagem & & & & & - & - & - & 2 & 2 & 2 & 6 & 7 \\
\hline Revista Gaúcha de Enfermagem & - & - & - & - & 1 & - & 3 & 1 & - & - & 5 & 6 \\
\hline Enfoque & 1 & 1 & - & - & - & - & - & - & - & - & 2 & 2 \\
\hline Texto e Contexto & & & & 2 & - & - & - & - & - & - & 2 & 2 \\
\hline Cogitare & & & & & & & & 1 & - & - & 1 & 1 \\
\hline Nursing & & & & & & & & - & - & 1 & 1 & 1 \\
\hline Total & 2 & 2 & 1 & 3 & $\mathbf{8}$ & 5 & 13 & 18 & 17 & 16 & 85 & 100 \\
\hline
\end{tabular}

A Revista Brasileira de Enfermagem é considerada a mais tradicional, a mais antiga e de maior divulgação entre a classe dos enfermeiros $^{(11-12)}$ e atende a demanda de todo o país. Mesmo assim, esta Revista, juntamente com a revista da Escola de Enfermagem da USP, constitui também um dos principais meios de comunicação e divulgação para os enfermeiros do Estado de Minas Gerais, como identificado na Tabela 2. Foram detectadas seis (7\%) publicações de enfermeiros mineiros na Revista Latino-americana de Enfermagem, periódico criado em 1993.

Verificamos que em 1993 iniciou-se um aumento na divulgação científica. Este fato talvez seja justificado pela criação da Revista de Enfermagem PUC/MG e do curso de Mestrado na Escola de Enfermagem da UFMG em 1994, fatos que sem dúvida estimulam a produção e publicação de trabalhos científicos. A partir de 1996, a divulgação científica tendeu a uma estabilidade.

Para analisar as publicações foi necessária a leitura de todos os textos, uma vez que os resumos não continham elementos suficientes para a classificação e análise dos trabalhos. Quando não estava explicitado no texto o tipo de trabalho, utilizamos conceito ou critério para classificá-los. Consideramos artigo científico um estudo completo, que trata de uma questão verdadeiramente científica ${ }^{(3)} \mathrm{e}$ foi também incluído na categoria de artigo científico os trabalhos de atualização e revisão, nesta categoria foram classificados a maioria $(53 \%)$ dos trabalhos analisados. Para a classificação de pesquisas foi observada a descrição metodológica, encontramos 20 (24\%) publicações. Nos demais estava explicitado o tipo de trabalho: relatos de experiência com $12(14 \%)$, resumos de teses com $4(5 \%)$ e resumos de dissertações, também com 4 (5\%).

Encontramos apenas dois resumos de dissertações em 1993 e dois em 1997. Considerando que o Estado de Minas Gerais tem um curso de mestrado desde o ano de 1994, com defesas de dissertações a partir de 1996. Faz-se necessário estimular mestres e mestrandos para que, tão logo defendam suas dissertações, publiquem o resumo das mesmas.

É importante destacar também que, graças a um convênio firmado entre a Escola de Enfermagem da USP e a Escola de Enfermagem da UFMG, a partir de 1992 foram oferecidas duas turmas de doutorado para docentes da EEUFMG, com a finalidade de agilizar a qualificação de seu professorado. Somente neste programa 11 professores obtiveram o título de doutor. No entanto, encontramos apenas quatro resumos de teses publicados no ano de 1997.

Dos trabalhos divulgados, 58 (68\%) apresentam unitermos, 
30 (35\%) têm tabelas, 19 (22\%) ilustrações ou figuras, e 15 (17\%) anexos. Todas as publicações analisadas apresentavam referências bibliográficas. Quanto aos resumos, 55 (65\%) publicações possuíam resumos em português e em outras línguas; 22 (26\%) não possuíam resumos de forma alguma; e oito (9\%) possuíam resumos só em português. Embora atualmente estes itens constituam norma de revista, no período de estudo estes ainda não constituíam normas de algumas delas. $O$ que pode ser confirmado por estes resultados.

Das seis áreas temáticas a que mais apresentou trabalhos publicados foi a assistencial (37\%), seguida de trabalhos voltados para a área profissional (20\%).

$\mathrm{Na}$ distribuição anual da produção científica, a área assistencial prevaleceu em todos os anos, confirmando uma tendência da área. Os trabalhos publicados em 1995 e 1996 contemplam todas as áreas temáticas analisadas.

$\mathrm{Na}$ área assistencial, a maioria dos trabalhos (19\%) tem o enfoque terapêutico, incluindo-se nessa categoria trabalhos relativos a tratamentos em geral, no âmbito hospitalar, desde que não enfatizassem os outros itens da assistência. 0 enfoque maternoinfantil (16\%) reuniu trabalhos sobre obstetrícia e recém-nascidos, e foi o segundo mais encontrado. 0 enfoque adolescência está equiparado com o de psiquiatria (10\%). No período estudado, não foram encontrados trabalhos sobre aspectos espirituais e psicológicos e nem nas diversas áreas hospitalares como hemodinâmica, CTI, bloco cirúrgico e outros.

A área profissional foi a segunda mais encontrada neste estudo, com $16(20 \%)$ trabalhos. Isto nos leva a supor que esteja havendo um maior engajamento dos profissionais nas discussões de aspectos das políticas de saúde e participação mais efetiva em órgãos de classe ou decisórios, além de uma maior preocupação em analisar a enfermagem enquanto prática social.

Dos 16 trabalhos da área profissional, 5 (31\%) referem-se a recursos humanos, seguidos de divulgação e utilização do conhecimento de enfermagem e autonomia profissional, com 3 (19\%) em cada uma delas; divisão social do trabalho, com 2 (13\%); e os demais referem-se a integração docente-assistencial, enfermagem e políticas de saúde, e ética e legislação. No período estudado não foram encontrados publicações sobre mercado de trabalho.

A maioria (87\%) das publicações da área de ensino focaliza a graduação e duas (13\%) a especialização. Foram os enfermeiros docentes que mais publicaram no período; no entanto, somente 15 (18\%) trabalhos estão relacionados com a área de ensino.

Dos trabalhos analisados apenas 5 (6\%) foram classificados na temática administração, no entanto, esta é a função mais valorizada e mais realizada pelos enfermeiros ${ }^{(11)}$.

Dos 85 trabalhos analisados, dois (2\%) dizem respeito à testagem de teorias, sendo esta a área menos encontrada no período estudado. Não foram encontrados trabalhos sobre elaboração e testagem de instrumentos e desenvolvimento de teorias.

Dos textos analisados, 16 (19\%) foram agrupados na categoria outros, por não se enquadrarem na classificação utilizada neste estudo. Conseqüentemente, os enfoques desses trabalhos eram os mais variados como: contexto social e sociedade, filosófico, visão de mundo e outros. Os resultados sugerem que o curso de mestrado, criado em 1994 na EEUFMG, pode ser um dos responsáveis pelo crescimento e diversidade das publicações da enfermagem.

Como a ocupação dos autores se diferenciou de um texto para outro, existe uma diferença entre o número de autores analisados (53) e as ocupações encontradas (57). Nos casos em que foram citados vários cargos, optou-se pelo primeiro cargo ou função, ou pela menção mais esclarecedora. A análise dos dados foi feita com o total de ocupações encontradas.

Há predominância de enfermeiros docentes $(75 \%)$, resultado já encontrado em outros estudos ${ }^{(10-13)}$ o que demonstra que é no seio da universidade que a produção e publicação são mais desenvolvidas.

Os autores assistenciais são dez (18\%), o que constata que a produção oriunda dos enfermeiros de campo ainda é sensivelmente pequena. Trata-se, no entanto, de uma contradição, pois é no próprio processo de trabalho, ou seja, no cuidado com o paciente, que se dá a essência da enfermagem. Alguns autores ${ }^{(13-}$ ${ }^{14)}$, justificam a pequena publicação da produção dos enfermeiros assistenciais pela dificuldade na elaboração de pesquisas, o desconhecimento do método científico, a falta de orientador, várias jornadas de trabalho culminando com a falta de tempo, falta de auxílio financeiro pelo desconhecimento da existência dos órgãos financiadores, falta de material bibliográfico disponível nas instituições e dificuldades no uso de computadores.

Detectamos apenas três (5\%) publicações de estudantes, o que demonstra que os mesmos ainda estão pouco envolvidos com a divulgação de estudos como autores.

No que se refere instituição a que pertence 0 autor, enfrentamos também dificuldade para a computação e análise dos dados, pois encontramos um mesmo autor publicando trabalhos e atuando em instituições distintas. Por isso, optamos por analisar essa variável levando em conta o total de publicações. Verificamos uma predominância de publicação de autores vinculados a instituições públicas, sendo a maioria $(72 \%)$ deles docentes.

Essa ocorrência pode ser atribuída, às funções exercidas pelos docentes nas instituições de ensino e à necessidade imposta pela universidade de ascensão na carreira universitária, o que requer a elaboração e publicação de trabalhos de pesquisa ${ }^{(14)}$. A própria academia é um fator que não só propicia o trabalho científico, como também valoriza estas produções. 
Para a classificação dos trabalhos segundo a titulação do autor, houve uma dificuldade ainda maior, uma vez que, ao longo do período estudado, um mesmo profissional pode ter publicado trabalhos nos diversos níveis de titulação, ou seja, graduado, especialista, mestrando, mestre, doutorando e doutor. Assim, a análise da titulação também foi feita com o número de publicação, e não com o número de autores.

Os graduados são número mais expressivo (33\%) de trabalhos científicos divulgados, com média de publicação de 1,2 trabalhos por autor. Os mestrandos publicaram igualmente aos mestres. Este fato pode ser justificado pela exigência de trabalhos nas disciplinas do curso de mestrado e com o incentivo à publicação ${ }^{(15)}$.

Os doutores e doutorandos apresentaram o mesmo número de trabalhos publicados no período. Embora o número de trabalhos seja menor que o dos graduados e mestres, a média de publicação dos doutores foi maior $(1,3)$. Cada um dos quatro especialistas publicou um trabalho e as três publicações sem informação sobre a titulação apresentaram também uma média de 1 por autor.

Encontramos 52 (62\%) trabalhos publicados com co-autoria. O número de co-autores variou de $1 \mathrm{a} 7$, porém a maioria dos trabalhos (90\%) tinha de 1 a 3 co-autores. Este fato levamos a considerar que existe uma tendência para a realização de trabalhos em grupos e este fato é importante para o desenvolvimento da produção científica da enfermagem ${ }^{(14)}$. No entanto, a análise foi feita com apenas 0 primeiro co-autor.

Com relação à ocupação dos co-autores, também verificamos que a maioria ( $83 \%$ ) é docente, seguidos de enfermeiros assistenciais (12\%) e graduandos (6\%). Com relação à titulação, observamos que a maioria dos co-autores é constituída de mestrandos $(27 \%)$, seguidos de graduados $(25 \%)$ e doutorandos $(15 \%)$.

\section{SINGULARIDADES}

Em relação aos 52 trabalhos com co-autoria, tivemos a diligência de saber "quem produz e publica com quem". Pudemos constatar que docentes produzem e publicam mais freqüentemente com colegas de trabalho e pouco com enfermeiros assistenciais e estudantes. Acreditamos que estes resultados sejam justificados pela atividade desenvolvida, ou seja, docentes do mesmo departamento ou integrantes do mesmo grupo de pesquisa.

De modo geral, os mestrandos como autores produzem e divulgam com co-autores de quase todas as titulações da academia. Neste estudo, os mesmos interagem mais com os colegas também mestrandos (14\%). De forma semelhante, os doutorandos produzem com doutorandos (10\%). Supomos que também seja pelas atividades de grupos dos cursos de mestrado e doutorado, onde as disciplinas exigem trabalhos e os resultados destes são publicados. Os doutores produzem e divulgam mais com doutores (8\%). Os graduados enquanto autores produzem e divulgam quase que exclusivamente com graduados $(5 \%)$.

Acreditamos que a pesquisa deve fazer parte do cotidiano profissional e pode envolver tanto o enfermeiro assistente, como 0 graduando ${ }^{(15)}$. Assim, a participação em grupos de pesquisa ou em realizações independentes devem ser estimuladas.

\section{CONSIDERAÇÕES FINAIS}

O estudo desenvolvido nos possibilitou apresentar uma visão preliminar da produção científica dos enfermeiros mineiros. Mesmo diante da sua limitação, os resultados oferecem subsídios para reflexão e estímulos para publicação e divulgação, assim como para estudos posteriores sobre o tema.

Os resultados encontrados não permitem estimativas abrangentes. No entanto, tendo em vista o número de enfermeiros cadastrados no COREn/MG, 5.613 até agosto de 1998 e o número das publicações (87) encontradas nos periódicos, podemos inferir que a freqüência de publicações ainda é tímida. $A$ área temática assistencial foi a mais encontrada no período de estudo com enfoque terapêutico.

A produção científica começa a expandir-se a partir de 1995, o que nos leva a inferir que a criação do mestrado na Escola de Enfermagem da UFMG possa ter uma influência neste resultado.

Diante do diagnóstico da situação da publicação científica da enfermagem mineira, sugerimos que haja maior valorização da publicação na graduação e pós-graduação; quer seja estudadas estratégias, nos currículos, para estimular a produção científica e sua publicação. Acreditamos também que se conhecermos melhor 0 que motiva os estudantes, suas potencialidades e principalmente sua criatividade, as escolas de Enfermagem poderiam interferir com mais eficácia na produção do conhecimento e sua divulgação. Os enfermeiros assistenciais devem ser mais estimulados pelas instituições para que relatem suas experiências, pesquisem e publiquem e as instituições devem valorizar nos planos de carreiras estas atividades profissionais e criar condições para que estes profissionais contribuam com a comunidade científica. Considerando que este trabalho foi do tipo exploratório, retrospectivo, limitada a publicações das revistas indexadas no Banco de Dados de Enfermagem, reafirmamos que o tema merece maiores investigações por parte dos pesquisadores, pois acreditamos que este trabalho serve de parâmetro para acompanhar a evolução da divulgação dos enfermeiros do Estado de Minas Gerais. 


\section{REFERÊNCIAS BIBLIOGRÁFICAS}

\section{Urbano CC, Oliveira CM, Coelho ES, Alves IKF, Silva LF, Mota SP} et al. Ser bolsista de Iniciação Científica: Relato de experiência. Rev Baiana Enfermagem 1995 abril/outubro; 8(1/2): 191-6.

2. Cooney JP. Qual o real valor da informação? Rev Esc Biblioteconomia, Belo Horizonte 1991 julho/dezembro; 20(2): 17690.

3. Lakatos Em, Marconi MA. Metodologia do trabalho científico. São Paulo: Atlas; 1992.

4. Silva EMP. Conhecimento e produção: peculiaridades da informação em ciência e tecnologia. Rev Esc Biblioteconomia UFMG 1992 julho/dezembro; 21(2):195-202.

5. Sadigursky D, Hurst IH, Tavares JL, Fernandes JD, Oliveira MRO. Pesquisa em enfermagem psiquiátrica: concepções e expectativa de enfermeiros que atuam em instituições psiquiátricas. Rev Latino-am Enfermagem 1998 dezembro; 6(5):89-98.

6. King D, Barnard KE, Hoehn R. Disseminating the results of nursing research. Nurs Outlook 1981 março; 29(3):164-9.

7. Müeller SPM. O impacto das tecnologias de informação na geração do artigo científico: tópicos para estudo. Rev Ciência Inf. 1994 setembro-dezembro; 23(3):309-17.
8. Assis MMA, Nascimento MAA, Jorge MSB, Matos MS, Braga VAB, Moreira ASP et al. Produção científica de enfermagem na Região Nordeste (1988-1992). Rev Latino-am Enfermagem 1993 dezembro; 1(número especial): 85-102.

9. Tsunechiro MA, Carvalho DV, Posso MBS, Elsas BX, Lui MC, Stefanelli MC. Instrumento para análise de periódico de enfermagem. Rev Esc Enfermagem USP 1983 maio; 17(2):107-17.

10. Carvalho EC. A produção do conhecimento de enfermagem. Rev Latino-am Enfermagem 1998 janeiro; 6(1):119-22.

11. Freitas DMV, Ruffino MC, Saeki T. A produção científica do enfermeiro do Estado de São Paulo no triênio 1985-1988. Rev Paul Enfermagem 1992 setembro-dezembro; 11(3):123-9.

12. Nachion MM, Carvalho EC, Stuchi RG. Retrospectiva da produção científica do SIBRACEn:1988-1994. Rev Latino-am Enfermagem 1998 abril; 6(2):47-55.

13. Cano MAT, Ferriani MGC, Alves AC, Nakata CY. A produção do conhecimento sobre adolescência na enfermagem: período 1983 a 1996. Rev Latino-am Enfermagem 1998 janeiro; 6(1):91-7.

14. Enoki H, Ferraz AEP, Carvalho EC, Marziale MHP. A produção científica acerca da comunicação em enfermagem. Rev Bras Enfermagem 1987 janeiro-março; 40(1):34-7.

15. ECHER IC, Crosseti MGO, Paskulin LMG, Cogo ALP, Santos VBD, Sottomaior VS et al. Criação da comissão de pesquisa do grupo de enfermagem do hospital de clínicas de Porto Alegre. Rev Gaúch Enfermagem 1998 janeiro; 19(1):56-9. 